\title{
New Upper Limit on the Total Neutrino Mass from the 2 Degree Field Galaxy Redshift Survey
}

\author{
Ø. Elgarøy, ${ }^{1}$ O. Lahav, ${ }^{1}$ W. J. Percival, ${ }^{2}$ J. A. Peacock, ${ }^{2}$ D. S. Madgwick, ${ }^{1}$ S. L. Bridle, ${ }^{1}$ C. M. Baugh, ${ }^{3}$ I. K. Baldry, ${ }^{4}$
} J. Bland-Hawthorn, ${ }^{5}$ T. Bridges, ${ }^{5}$ R. Cannon, ${ }^{5}$ S. Cole,${ }^{3}$ M. Colless, ${ }^{6}$ C. Collins, ${ }^{7}$ W. Couch, ${ }^{8}$ G. Dalton, ${ }^{9}$ R. De Propris, ${ }^{8}$ S. P. Driver, ${ }^{10}$ G. P. Efstathiou, ${ }^{1}$ R. S. Ellis, ${ }^{11}$ C. S. Frenk, ${ }^{3}$ K. Glazebrook,${ }^{5}$ C. Jackson, ${ }^{6}$ I. Lewis, ${ }^{9}$ S. Lumsden, ${ }^{12}$ S. Maddox, ${ }^{13}$ P. Norberg, ${ }^{3}$ B. A. Peterson, ${ }^{6}$ W. Sutherland, ${ }^{2}$ and K. Taylor ${ }^{11}$

${ }^{1}$ Institute of Astronomy, University of Cambridge, Madingley Road, Cambridge CB3 OHA, United Kingdom ${ }^{2}$ Institute for Astronomy, University of Edinburgh, Royal Observatory, Blackford Hill, Edingburgh EH9 3HJ, United Kingdom

${ }^{3}$ Department of Physics, University of Durham, South Road, Durham DH1 3LE, United Kingdom

${ }^{4}$ Department of Physics \& Astronomy, Johns Hopkins University, Baltimore, Maryland 21218-2686

${ }^{5}$ Anglo-Australian Observatory, P.O. Box 296, Epping, New South Wales 2121, Australia

${ }^{6}$ Research School of Astronomy \& Astrophysics, The Australian National University, Weston Creek, ACT 2611, Australia

${ }^{7}$ Astrophysics Research Institute, Liverpool John Moores University, Twelve Quays House, Birkenhead L14 1LD, United Kingdom

${ }^{8}$ Department of Astrophysics, University of New South Wales, Sydney, New South Wales 2052, Australia

${ }^{9}$ Department of Physics, University of Oxford, Keble Road, Oxford OX1 3RH, United Kingdom

${ }^{10}$ School of Physics and Astronomy, University of St. Andrews, North Haugh, St. Andrews, Fife KY6 9SS, United Kingdom

${ }^{11}$ Department of Astronomy, California Institute of Technology, Pasadena, California 91125

${ }^{12}$ Department of Physics, University of Leeds, Woodhouse Lane, Leeds LS2 9JT, United Kingdom

${ }^{13}$ School of Physics \& Astronomy, University of Nottingham, Nottingham NG7 2RD, United Kingdom (Received 9 April 2002; published 19 July 2002)

We constrain $f_{\nu} \equiv \Omega_{\nu} / \Omega_{\mathrm{m}}$, the fractional contribution of neutrinos to the total mass density in the Universe, by comparing the power spectrum of fluctuations derived from the 2 Degree Field Galaxy Redshift Survey with power spectra for models with four components: baryons, cold dark matter, massive neutrinos, and a cosmological constant. Adding constraints from independent cosmological probes we find $f_{\nu}<0.13$ (at $95 \%$ confidence) for a prior of $0.1<\Omega_{\mathrm{m}}<0.5$, and assuming the scalar spectral index $n=1$. This translates to an upper limit on the total neutrino mass $m_{\nu \text {,tot }}<1.8 \mathrm{eV}$ for "concordance" values of $\Omega_{\mathrm{m}}$ and the Hubble constant.

DOI: 10.1103/PhysRevLett.89.061301

Whether neutrinos are massive or not has been an open question for a long time, but the recent data from atmospheric and solar neutrino experiments [1-7] are most naturally interpreted in terms of neutrino oscillations, which implies that not all neutrinos are massless [8]. However, since the oscillation probability depends on the mass-squared differences, and not on the absolute masses, the oscillation experiments cannot provide absolute masses for neutrinos. The mass scale can, in principle, be obtained from, e.g., the energy spectrum in the beta decay of ${ }^{3} \mathrm{H}$ [9], or from neutrinoless double beta decay $[10,11]$. At the present, cosmological data [12-14] provide stronger constraints on the total neutrino mass than particle physics experiments. Since neutrinos with masses of the order of a few tenths of an electron volt $(\mathrm{eV})$ can have a significant effect on the formation of large-scale structures in the Universe, observations of the distribution of galaxies can provide us with an upper bound on the density of massive neutrinos. We will in this paper use data from the 2 Degree Field Galaxy Redshift Survey (2dFGRS), which is the largest existing redshift survey $[15,16]$, to obtain an upper bound on the sum of the neutrino masses.

Massive neutrinos make up part of the dark matter in the Universe. In the cosmological model favored by data on large-scale structure and the observed fluctuations in the cosmic microwave background (CMB) $[17,18]$, the
PACS numbers: 95.35.+d, 14.60.Pq, 98.62.Py, 98.80.Es

Universe is flat, and the contributions to the mass-energy density in units of the critical density are $\Omega_{\Lambda} \approx 0.7$ from vacuum energy or a "quintessence" field, and $\Omega_{\mathrm{m}} \approx 0.3$ from matter. Baryons make up only $f_{\mathrm{b}} \equiv \Omega_{\mathrm{b}} / \Omega_{\mathrm{m}} \approx 0.15$ of the matter contribution $[17,18]$, most of the remaining being in the form of cold dark matter (CDM), the exact nature of which is still unknown. "Cold" in this context means that the particles were moving at nonrelativistic speeds when they dropped out of thermal equilibrium. Particles drop out of equilibrium roughly when their interaction rate falls below the expansion rate of the Universe. For neutrinos with masses in the $\mathrm{eV}$ range this happened when they were still relativistic, and so they will be a "hot" component of the dark matter. This has implications for large-scale structure, since the neutrinos can free-stream over large distances and erase small-scale structures (see, e.g., [19] for an overview). As a result, mass fluctuations are suppressed at comoving wave numbers greater than $k_{\mathrm{nr}}=0.026\left(m_{\nu} / 1 \mathrm{eV}\right)^{1 / 2} \Omega_{\mathrm{m}}^{1 / 2} h \mathrm{Mpc}^{-1}$ [20], where $m_{\nu}$ is the neutrino mass of one flavor. The neutrino contribution to the total mass-energy density, $\Omega_{\nu}$, in units of the critical density needed to close the Universe, is given by

$$
\Omega_{\nu} h^{2}=\frac{m_{\nu, \mathrm{tot}}}{94 \mathrm{eV}}
$$

where $m_{\nu, \text { tot }}$ is the sum of the neutrino mass matrix eigen- 
values, and the Hubble parameter $H_{0}$ at the present epoch is given in terms of $h$ as $H_{0}=100 h \mathrm{~km} \mathrm{~s}^{-1} \mathrm{Mpc}^{-1}$. Equation (1) assumes that all three neutrino flavors drop out of equilibrium at the same temperature, which is a reasonable approximation. The suppression of the matter power spectrum $P_{\mathrm{m}}(k)$ on small scales is given approximately by (see, e.g., [21]) $\Delta P_{\mathrm{m}} / P_{\mathrm{m}} \approx-8 f_{\nu}$, where $f_{\nu} \equiv$ $\Omega_{\nu} / \Omega_{\mathrm{m}}$. Therefore even a neutrino mass as small as $0.1 \mathrm{eV}$ gives a reduction in power of $5 \%-15 \%$.

The 2dFGRS has now measured over 220000 galaxy redshifts, with a median redshift of $z_{\mathrm{m}} \approx 0.11$, and is the largest existing galaxy redshift survey [15]. A sample of this size allows large-scale structure statistics to be measured with very small random errors. An initial estimate of the convolved, redshift-space power spectrum of the 2dFGRS has been determined [16] for a sample of 160000 redshifts. On scales $0.02<k<0.15 h \mathrm{Mpc}^{-1}$, the data are robust and the shape of the power spectrum is not affected by redshift-space or nonlinear effects, though the amplitude is increased by redshift-space distortions. These data and their associated covariance matrix form the basis for our analysis.

For each model, we calculate its linear-theory matter power spectrum, and for the $2 \mathrm{dFGRS} \mathrm{power} \mathrm{spectrum} \mathrm{data}$ it is sufficiently accurate to use the fitting formulas derived in [22]. The relation between the measured galaxy power spectrum and the calculated matter power spectrum is given by the so-called bias parameter $b^{2} \equiv P_{g}(k) / P_{m}(k)$. By definition, $b$ is, in principle, a function of scale, and several biasing scenarios have been proposed [23]. This issue is of some importance for our analysis. For example, if the galaxy distribution is more biased on small scales than on large scales, a nonzero best-fit value for $f_{\nu}$ may be obtained. However, on the scales we consider there are theoretical reasons to expect that $b$ should tend to a constant [24]. For the 2dFGRS, a recent analysis [25] looking for deviations from linear biasing found no evidence for it. We will therefore in the following assume that the biasing is scale independent. In particular, two independent analyses $[25,26]$ suggest that the data are consistent with $b \approx 1$ on large scales. We choose to avoid the complications in the normalization of the power spectra caused by redshiftspace distortions and the actual value of the bias parameter by leaving the amplitude of the power spectrum, from here on denoted by $A$, as a free parameter, and then marginalize over it.

We shall consider here a model with four components: baryons, cold dark matter, massive neutrinos (hot dark matter), and a cosmological constant. As an illustration, we show in Fig. 1 the power spectra for $\Omega_{\nu}=0,0.01$, and 0.05 (all other parameters are fixed at their "concordance model" values given in the figure caption), after they havebeen convolved with the 2dFGRS window function, and their amplitudes fitted to the 2dFGRS power spectrum data. For the 32 data points, the $\Omega_{\nu}=0$ model had $\chi^{2}=$ 32.9, $\Omega_{\nu}=0.01$ gives $\chi^{2}=33.4$, whereas the model with $\Omega_{\nu}=0.05$ provides a poor fit to the data with $\chi^{2}=92.2$.

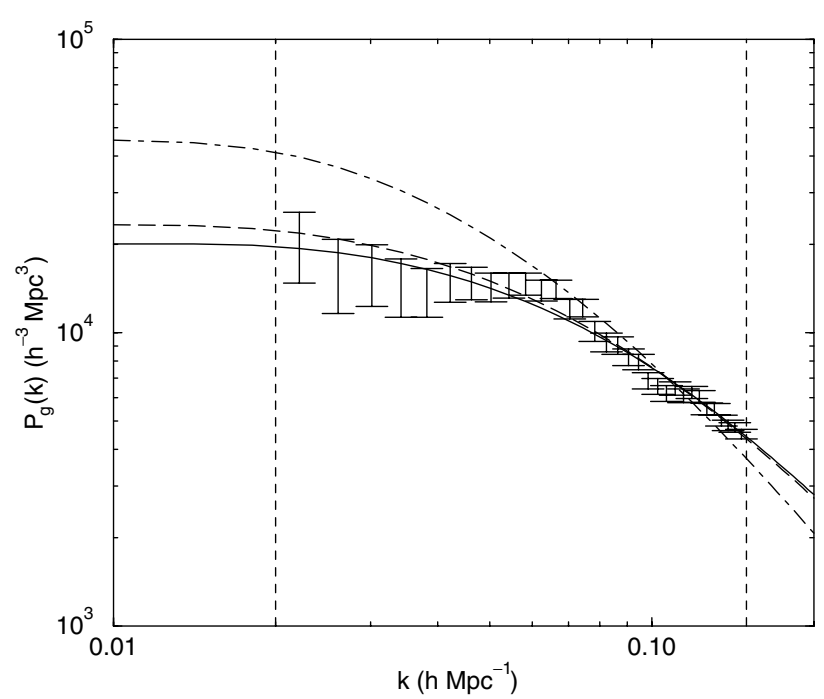

FIG. 1. Power spectra for $\Omega_{\nu}=0$ (solid line), $\Omega_{\nu}=0.01$ (dashed line), and $\Omega_{\nu}=0.05$ (dot-dashed line) with amplitudes fitted to the 2dFGRS power spectrum data (vertical bars) in redshift space. We have fixed $\Omega_{\mathrm{m}}=0.3, \Omega_{\Lambda}=0.7, h=0.7$, and $\Omega_{\mathrm{b}} h^{2}=0.02$. The vertical dashed lines limit the range in $k$ used in the fits.

Clearly, the inference of the neutrino mass depends on our assumptions ("priors") on the other parameters. We therefore add constraints from other independent cosmological probes. The Hubble parameter has been determined by the HST Hubble key project to be $h=0.70 \pm 0.07$ [27], and big bang nucleosynthesis gives a constraint $\Omega_{\mathrm{b}} h^{2}=$ $0.020 \pm 0.002$ on the baryon density [28]. For these parameters, we adopt Gaussian priors with the standard deviations given above.

Perhaps the least known prior is the total matter density $\Omega_{\mathrm{m}}$. The position of the first peak in the CMB power spectrum gives a strong indication that the Universe is spatially flat, i.e., $\Omega_{\mathrm{m}}+\Omega_{\Lambda}=1[17,18]$. The CMB peak positions are not sensitive to neutrino masses, because the neutrinos were nonrelativistic at recombination, and hence indistinguishable from cold dark matter. Although the shape of the power spectrum is independent of curvature, the curvature does affect the choice of priors on $\Omega_{\mathrm{m}}$, and we choose to consider flat models only. When the constraint of a flat universe is combined with surveys of high redshift Type Ia supernovae [29,30], one finds $\Omega_{\mathrm{m}}=$ $0.28 \pm 0.14$. However, studies of the mass-to-light ratio of galaxy clusters find values of $\Omega_{\mathrm{m}}$ as low as 0.15 [31], whereas cluster abundances give a range of values $\Omega_{\mathrm{m}} \approx$ $0.3-0.9$ [32-37]. We will therefore use two different priors on $\Omega_{\mathrm{m}}$. The first is a Gaussian centered at $\Omega_{\mathrm{m}}=0.28$ with standard deviation 0.14, motivated by [29]. As an alternative, we use a uniform ("top hat") prior in the range $0.1<\Omega_{\mathrm{m}}<0.5$. Given that we use the HST Key Project result [27] for $h, \Omega_{\mathrm{m}}<0.5$ is required to be consistent with the age of the Universe [38] being greater than 12 Gyr.

Finally, the CMB data $[18,39,40]$ are consistent with the scalar spectral index of the primordial power spectrum 

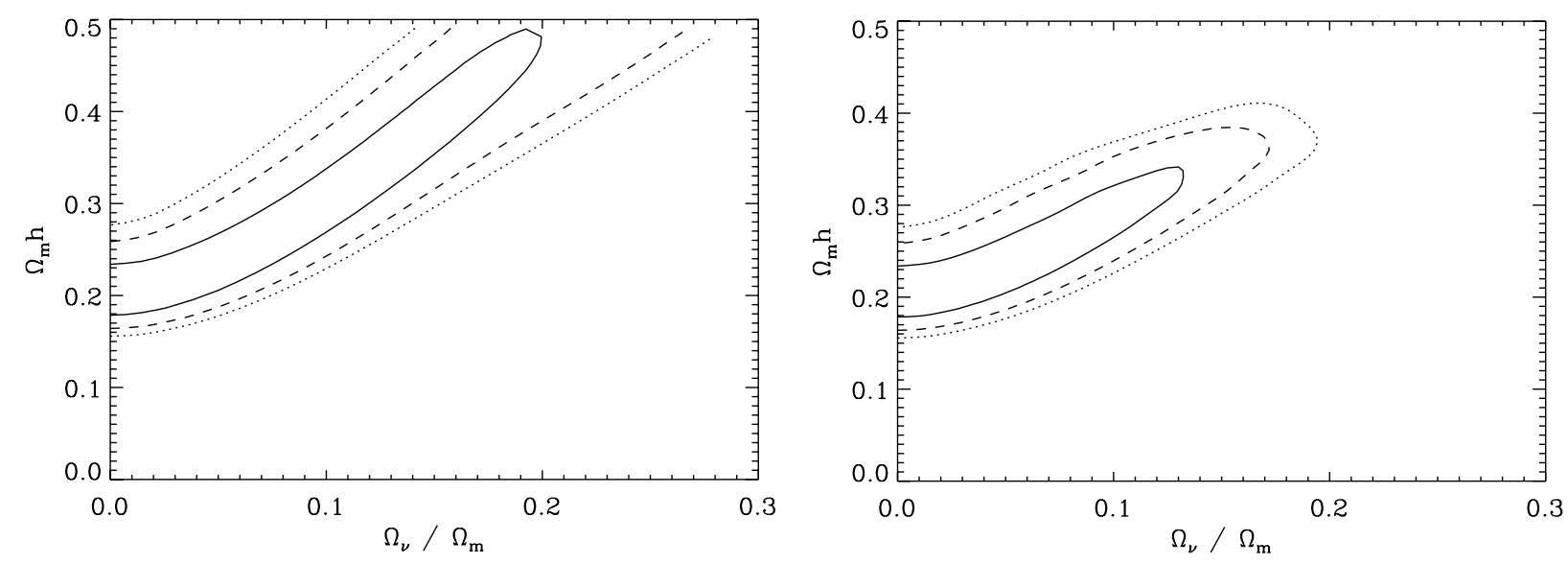

FIG. 2. Confidence contours at $68 \%$ (solid line), $95 \%$ (dashed line), and $99 \%$ (dotted line) in the plane of $f_{\nu} \equiv \Omega_{\nu} / \Omega_{\mathrm{m}}$ and $\Gamma \equiv$ $\Omega_{\mathrm{m}} h$, with marginalization over $h$ and $\Omega_{\mathrm{b}} h^{2}$ using Gaussian priors, and over $A$ using a uniform prior in $0.5<A<10$. The left panel shows the case of no prior on $\Omega_{\mathrm{m}}$, and the right panel the case of a uniform "top hat" prior on $\Omega_{\mathrm{m}}$ in $0.1<\Omega_{\mathrm{m}}<0.5$.

being $n=1$, but we also quote results for $n=0.9$ and $n=$ 1.1 , and for the case when we marginalize over $n$ with a Gaussian prior $n=1.0 \pm 0.1$, motivated by the latest data from VSA and CBI $[39,40]$.

Results will be presented for the case of $N_{\nu}=3$ equalmass neutrinos, but the derived upper bound on the total neutrino mass is only marginally different for $N_{\nu}=1$ or 2 . For each set of parameters, we computed the theoretical matter power spectrum, and obtained the $\chi^{2}$ for the model given the 2dFGRS power spectrum. We then calculated the joint probability distribution function for $f_{\nu}$ and $\Gamma \equiv \Omega_{\mathrm{m}} h$ (which represents the shape of the CDM power spectrum) by marginalizing over $A, h$, and $f_{\mathrm{b}}$ weighted by the priors given above. For $A$ we used a uniform prior in the interval $0.5<A<10$, where $A=1$ corresponds to the normalization of the "concordance model," discussed in [26]. Using instead a prior uniform in $\log A$, or fixing $A$ at the best-fit value had virtually no effect on the results. We evaluated the likelihood on a grid with $0.1<\Omega_{\mathrm{m}} h<0.5$, $0 \leq f_{\nu}<0.3,0<f_{\mathrm{b}}<0.3,0.4<h<0.9$, and $0.5<A<$ 10. In [18] it was found that the $2 \mathrm{dFGRS}$ data alone allow a solution with a high baryon density $f_{\mathrm{b}}=0.4$, in addition to a low density-low baryon density solution. However, given the above priors, the solution with high baryon density gets little weight and the fitting-formulas in [22] are sufficiently accurate for the measured BBN baryon density.

The results are shown in Fig. 2 for the cases of no prior on $\Omega_{\mathrm{m}}$ (left panel) and with the uniform prior $0.1<\Omega_{\mathrm{m}}<$ 0.5 (right panel). Marginalizing the distributions in the right panel of Fig. 2 over $\Omega_{\mathrm{m}} h$, we get the one-dimensional distribution for $f_{\nu}$ given by the solid line in Fig. 3, and an upper limit $f_{\nu}<0.13$ at $95 \%$ confidence. For comparison, marginalizing without any priors, the limit becomes $f_{\nu}<$ 0.24 . Adding just a prior on $\Omega_{\mathrm{m}}$, we find $f_{\nu}<0.15$, so this is clearly the most important prior. Marginalizing with just a prior on $h$ or on $\Omega_{\mathrm{b}} h^{2}$, the $95 \%$ confidence limit becomes $f_{\nu}<0.20$. As a further test of the stability of our analysis, we used the full set of priors, but only the power spectrum data at scales $k<0.1 h \mathrm{Mpc}^{-1}$. In this case the limit increases to $f_{\nu}<0.20$.

There is a further degeneracy of $f_{\nu}$ with the scalar spectral index $n$, since increasing $n$ increases power on small scales and leaves more room for suppression by the massive neutrinos. Also shown in Fig. 3 are the distributions for the cases $n=0.9$ (dotted line) and $n=1.1$ (dashed line). With $n=1.1$, the $95 \%$ confidence limit on $f_{\nu}$ increases slightly to 0.16 . The results are summarized in Fig. 3 and in Table I. Also included in this table are the results obtained using the Type Ia supernova prior, and it is seen that the results for the two different choices are almost

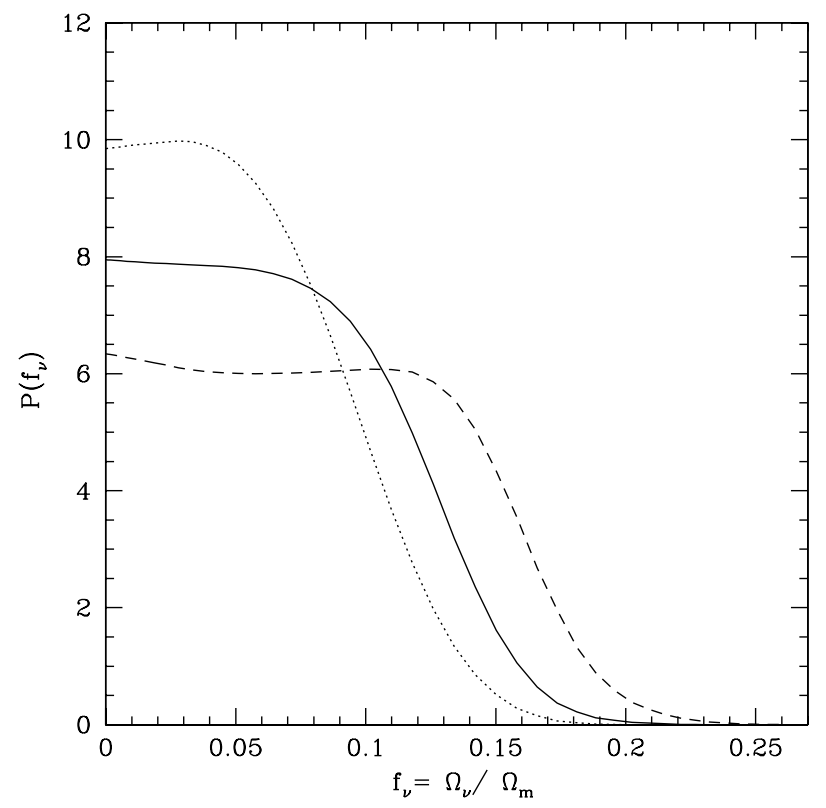

FIG. 3. Probability distributions, normalized so that the area under each curve is equal to 1 , for $f_{\nu}$ with marginalization over the other parameters, as explained in the text, for $N_{\nu}=3$ massive neutrinos and $n=0.9$ (dotted line), 1.0 (solid line), and 1.1 (dashed line). 
TABLE I. Summary of $95 \%$ confidence upper bounds on $f_{\nu}$ with our two chosen priors on $\Omega_{\mathrm{m}}$. The conversion of $f_{\nu}$ to $m_{\nu \text {,tot }}$ is for $h=0.7$ and the central values of $\Omega_{\mathrm{m}}=0.28$ (SNIa case) and $\Omega_{\mathrm{m}}=0.30$ (uniform prior case).

\begin{tabular}{ccccc}
\hline \hline & \multicolumn{2}{c}{$\Omega_{\mathrm{m}}=0.28 \pm 0.14(\mathrm{SNIa})$} & \multicolumn{2}{c}{$0.1<\Omega_{\mathrm{m}}<0.5$} \\
$n$ & $f_{\nu}$ & $m_{\nu, \text { tot }}(\mathrm{eV})$ & $f_{\nu}$ & $m_{\nu, \text { tot }}(\mathrm{eV})$ \\
\hline 0.9 & 0.12 & 1.5 & 0.11 & 1.5 \\
1.0 & 0.14 & 1.8 & 0.13 & 1.8 \\
1.1 & 0.16 & 2.1 & 0.16 & 2.2 \\
\hline \hline
\end{tabular}

identical. Running a grid of models with $n$ as an added parameter, and marginalizing with a prior $n=1.0 \pm 0.1$, consistent with the CMB data $[39,40]$, we find $f_{\nu}<0.16$ at $95 \%$ confidence.

To summarize, we have analyzed the shape of the 2dFGRS power spectrum to obtain an upper bound on the fractional contribution of massive neutrinos to the total mass density, $f_{\nu}$, and found an upper limit $f_{\nu}<$ 0.13 at $95 \%$ confidence for $0.1<\Omega_{\mathrm{m}}<0.5$ and the scalar spectral index $n=1$. This translates into a constraint on the sum of the neutrino mass matrix eigenvalues $m_{\nu \text {,tot }}<$ $1.8 \mathrm{eV}$ for $\Omega_{\mathrm{m}} h^{2}=0.15$. With marginalization over $n$ with a prior $n=1.0 \pm 0.1$, the limit becomes $m_{\nu, \text { tot }}<2.2 \mathrm{eV}$. Previous cosmological bounds on neutrino masses come from data on galaxy cluster abundances [12,41], the Lyman- $\alpha$ forest [13], and compilations of data including the $\mathrm{CMB}$, peculiar velocities, and large-scale structure [14]. They give upper bounds on the total neutrino mass in the range 3-6 eV. Note that the fluctuation amplitude derived from the cluster abundances is still under some debate [32-35]. The most recent limit is that of [20]; from a combined analysis of CMB and large-scale structure data an upper limit $m_{\nu \text {,tot }}<4.2 \mathrm{eV}$ was found. Our bounds, summarized in Table I, are stronger, largely because of the small statistical errors in the 2dFGRS power spectrum, although the priors on the other parameters, in particular, on $\Omega_{\mathrm{m}}$, are also important. Note also that all of these results are stronger than current constraints from particle physics. As they stand, the controversial results of [11] imply a nearly degenerate neutrino mass matrix and a bound on neutrino mass matrix eigenvalue $0.1<m_{\nu}<1-20 \mathrm{eV}$ [42-44], where the upper limit is somewhat model dependent. Our results are consistent with this range. If the largest neutrino mass is, in fact, of order a tenth of an $\mathrm{eV}$, it should be possible to measure its value using a combination of cosmological data, combining 2dFGRS, SDSS, MAP, and/ or Planck.

$\varnothing$. E. acknowledges support from the Research Council of Norway.

[1] S. Fukuda et al., Phys. Rev. Lett. 85, 3999 (2000).

[2] M. Ambrosio et al., Phys. Lett. B 517, 59 (2001).

[3] B. T. Cleveland et al., Astrophys. J. 496, 505 (1998).

[4] The Gallex Collaboration, Phys. Lett. B 447, 127 (1999).
[5] J. N. Abdurashitov et al., Phys. Rev. C 60, 055801 (1999).

[6] M. Altmann et al., Phys. Lett. B 490, 16 (2000).

[7] Q. R. Ahmad et al., Phys. Rev. Lett. 87, 071301 (2001).

[8] J. N. Bahcall, M. C. Gonzalez-Garcia, and C. Pena-Garay, J. High Energy Phys. 0108, 14 (2001).

[9] J. Bonn et al., Nucl. Phys. Proc. Suppl. 91, 280 (2001).

[10] H. V. Klapdor-Kleingrothaus et al., Eur. Phys. J. A 12, 147 (2001).

[11] H. V. Klapdor-Kleingrothaus et al., Mod. Phys. Lett. A 37, 2409 (2001).

[12] M. Fukugita, G-C. Liu, and N. Sugiyama, Phys. Rev. Lett. 84, 1082 (2000).

[13] R. A. C. Croft, W. Hu, and R. Davé, Phys. Rev. Lett. 83, 1092 (1999).

[14] E. Gawiser and J. Silk, Science 280, 1405 (1998).

[15] M. Colless et al., Mon. Not. R. Astron. Soc. 328, 1039 (2001).

[16] W. J. Percival et al., Mon. Not. R. Astron. Soc. 327, 1297 (2001).

[17] G. P. Efstathiou et al., Mon. Not. R. Astron. Soc. 330, L29 (2002).

[18] X. Wang, M. Tegmark, and M. Zaldarriaga, Phys. Rev. D 65, 123001 (2002).

[19] J. R. Primack, astro-ph/0112255.

[20] A. G. Doroshkevich, Ya. B. Zel'sdovich, R. A. Syunyaev, and M. Yu. Khlopov, Sov. Astron. Lett. 6, 252 (1980).

[21] W. Hu, D. J. Eisenstein, and M. Tegmark, Phys. Rev. Lett. 80, 5255 (1998).

[22] D. J. Eisenstein and W. Hu, Astrophys. J. 511, 5 (1999).

[23] A. Dekel and O. Lahav, Astrophys. J. 520, 24 (1999).

[24] A. J. Benson, S. Cole, C. S. Frenk, C. M. Baugh, and C. G. Lacey, Mon. Not. R. Astron. Soc. 311, 793 (2000).

[25] L. Verde et al., Mon. Not. R. Astron. Soc. (to be published).

[26] O.Lahav et al., Mon. Not. R. Astron. Soc. 333, 961 (2002).

[27] W. L. Freedman et al., Astrophys. J. 553, 47 (2001).

[28] S. Burles, K. M. Nollett, and M. S. Turner, Phys. Rev. D 63, 063512 (2001)

[29] S. Perlmutter et al., Astrophys. J. 517, 565 (1999).

[30] A. G. Riess et al., Astrophys. J. 117, 707 (1999).

[31] N. A. Bahcall and J. M. Comerford, Astrophys. J. Lett. 565, L5 (2002).

[32] E. Pierpaoli, D. Scott, and M. White, Mon. Not. R. Astron. Soc. 325, 77 (2001).

[33] U. Seljak, astro-ph/0111362.

[34] T. H. Reiprich and H. Boehringer, Astrophys. J. 567, 716.

[35] P. T. P. Viana, R. C. Nichol, and A. R. Liddle, Astrophys. J. 569, L75 (2002).

[36] A. Blanchard, R. Sadat, J. G. Bartlett, and M. LeDour, Astron. Astrophys. 362, 809 (2000).

[37] V. R. Eke, S. Cole, C. S. Frenk, and J. P. Henry, Mon. Not. R. Astron. Soc. 298, 1145 (1998).

[38] L. M. Krauss and B. Chaboyer, astro-ph/0111597.

[39] J. A. Fubiño-Martin et al., astro-ph/0205367.

[40] J. L. Sievers et al., astro-ph/0205387.

[41] N. A. Arhipova, T. Kahniashvili, and V. N. Lukash, Astron. Astrophys. 386, 775 (2002).

[42] F. Feruglio, A. Strumia, and F. Vissani, Nucl. Phys. B (to be published).

[43] Z. Xing, Phys. Rev. D 65, 077302 (2002).

[44] V. Barger, S. L. Glashow, D. Marfiata, and K. Whisnant, Phys. Lett. B 532, 15 (2002). 